\title{
CONTROL DIFUSO PARA UNA PLANTA PILOTO DE SECADO POR LECHO FLUIDIZADO
}

\author{
Flavio Torres V. ${ }^{1} \quad$ Carlos Muñoz P. ${ }^{2} \quad$ Iván Velásquez B. ${ }^{3} \quad$ Carolina Shene V. \\ Recibido el 04 de junio de 2003, aceptada el 31 de diciembre de 2003.
}

\begin{abstract}
RESUMEN
En este trabajo se presenta un control difuso proporcional-prealimentado (CDPP) aplicado a un proceso de secado por lecho fluidizado de material húmedo. El CDPP se somete a diferentes experimentos y se compara ventajosamente con el control difuso proporcional-integral (CDPI). Los experimentos realizados permiten observar que el CDPP tiene un mejor comportamiento que el CDPI, ya que con él no se observan oscilaciones en la salida, aspecto clave para el correcto funcionamiento del proceso de secado por fluidización.
\end{abstract}

Palabras claves: Lecho fluidizado, control fuzzy, restricciones.

\begin{abstract}
In this work a fuzzy feedforward proportional control (FFPC) applied to a process of fluidized bed dryer of humid material is presented. The FFPC is submitted to different experiments and is adventagously compared with the fuzzy proportional-integral control (FPIC). The experiments show that the FFPC has a better behavior that the FPIC, since there is no evidence of output oscilations, key aspect for the correct functioning of the process of fluidized bed dryer.
\end{abstract}

Keywords: Fuidized bed, fuzzy inference, constraints

\section{INTRODUCCIÓN}

El secado por fluidización es una técnica para eliminar la humedad de un sólido mediante el paso de un gas, ó líquido, a través de un lecho donde se encuentra depositado el sólido. La velocidad del fluido debe ser tal que los sólidos "floten” en él. La aplicación de ésta técnica se encuentra en numerosos procesos físicos metalúrgicos y químicos [1], [2]. La principal variable a controlar es la temperatura del lecho.

En el proceso de fluidización es vital mantener la temperatura constante, preestablecida por un operador, durante todo el tiempo que dura el secado, por lo tanto se requiere un control de temperatura para neutralizar las perturbaciones (temperatura del medio ambiente, contenido de humedad de los materiales, etc.)

El Instituto de Agroindustrias de la Universidad de la Frontera de Chile posee un secador de lecho fluidizado al nivel de planta piloto que permite ensayar con diferentes materiales, principalmente de tipo afrecho. En este proceso los sólidos húmedos del lecho son secados por inyección de aire caliente A su vez el aire caliente se obtiene al hacer pasar aire del medio ambiente a través de una cámara de calefactores eléctricos. Por lo tanto, la temperatura al interior del lecho se gobierna regulando la potencia de los calefactores.

Los calefactores eléctricos funcionan sólo en estado $\mathrm{ON}$ y OFF. Bajo estas condiciones se probaron diferentes estrategias de control para gobernar la potencia de los calefactores. Buscando la simplicidad y el bajo costo, en Medina [3] se controló la temperatura al interior del lecho mediante un control ON/OFF de los calefactores. No obstante su simplicidad, este control produce oscilaciones excesivas en la temperatura del lecho debido al retardo inherente asociado a éste tipo de proceso, lo que impide alcanzar mayores índices de calidad requeridos para el producto final del proceso de secado.

En el presente trabajo se demuestra que se consigue una notable mejoría al cambiar el control ON/OFF por control continuo. Sin embargo para lograr lo anterior, sin modificar la actuación existente, se optó por emplear

\footnotetext{
1 Universidad de La Frontera, Departamento de Ingeniería Eléctrica, Casilla 54-D, Temuco, Chile. Fono (45) 325534. E-mail: ftorres@ufro.cl

2 Universidad de la Frontera, Dr. en ciencias de la Ingeniería, Pontificia Universidad Católica de Chile

3 Universidad Federico Santa María, Magister en Procesos Químicos, Universidad Federico Santa María.

4 Universidad de Santiago de Chile, Dr. en Ciencias de la Ingeniería, Universidad de Chile.
} 
la técnica de modulación de ancho de pulso (PWM), ya que no tiene mayores inconvenientes para trabajar en un lazo lento de control continuo de temperatura como el del lecho fluidizado. A continuación se presenta una alternativa al algoritmo PID para el control de temperatura del lecho fluidizado. Aunque el control PID es aplicable a este tipo de proceso, se demuestra que los algoritmos basados en técnicas difusas presentan una notable ventaja [4]. En este trabajo se aplica un control difuso proporcional prealimentado (CDPP) [1] al secador de lecho fluidizado y se comparan con un control difuso proporcional in tegral (CDPI).

\section{DESCRIPCIÓN DEL PROCESO}

El esquema de la planta de secado se muestra en la Fig. 1 y consta de: calefactores, cámara de secado, ciclón, regulador manual de flujo, extractor de aire, entrada del producto, salida del producto, filtro de aire y extractor de aire. Los calefactores consisten en un banco trifásico de resistencia de $6 \mathrm{Kw}$. y $4 \mathrm{Kw}$. de potencia. El ciclón permite la absorción de partículas finas que son arrastradas hacia el filtro por la corriente de aire y que posteriormente son decantadas. El regulador manual de flujo permite controlar la velocidad y caudal de aire que pasa por el sistema y que dependerá del producto a secar. El extractor de aire consiste de un motor trifásico de $1.5 \mathrm{Kw}$. en cuyo eje se acopla un ventilador.

La planta de secado está instrumentada con un PLC Siemens. El sensor de temperatura y el control de los calefactores se conectan al PLC. Una interfaz entre el PLC y un PC permite programar el PLC y comunicarlo con el software SCADA Intouch de Wonderware para manejar y supervisar la planta en forma amigable a través de despliegues gráficos.

Las Figs. 2, 3 y 4 muestran las curvas de reacción de temperatura al interior del lecho frente a escalones de potencia eléctrica en los calefactores de diferente magnitud cuando se secan 500 gramos de afrechillo (33\% de humedad). De las curvas se observa que tanto tiempo de subida como la ganancia obtenidos ante cambios en escalón de diferente magnitud aplicados a la entrada no son constantes. Al realizar repetidamente estos experimentos se observaron resultados similares, por lo que se dedujo que el fenómeno observado corresponde a una no linealidad inherente al proceso. Al repetir estas pruebas se constató que la no linealidad en la respuesta de temperatura se mantuvo, lo que amerita el empleo de técnicas difusas para el control de temperatura. Adicionalmente en la Fig. 5 se aprecia que incluso la relación en estado estacionario entre la potencia eléctrica y la temperatura al interior del lecho presenta una no linealidad fuerte.

La no linealidad observada, y el hecho de que en este proceso se requiere operar en diferentes valores de referencia dado que se trata de un proceso batch, sugirió la necesidad de abordar el diseño utilizando técnicas de control no lineal como el control difuso.

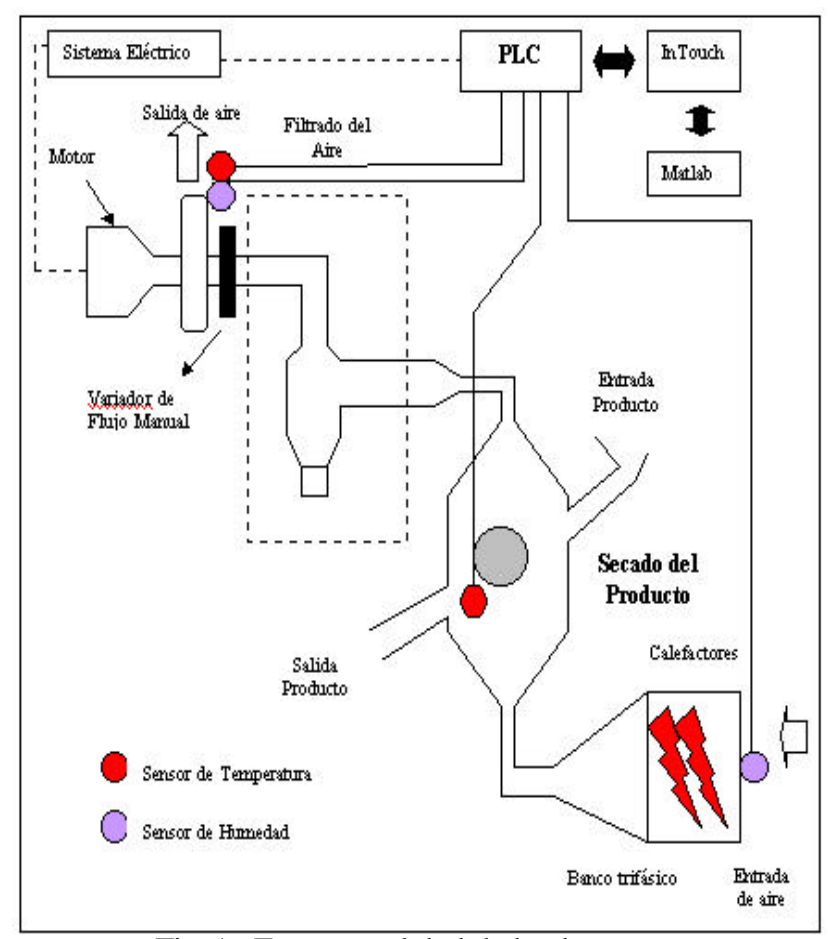

Fig. 1.- Esquema global de la planta

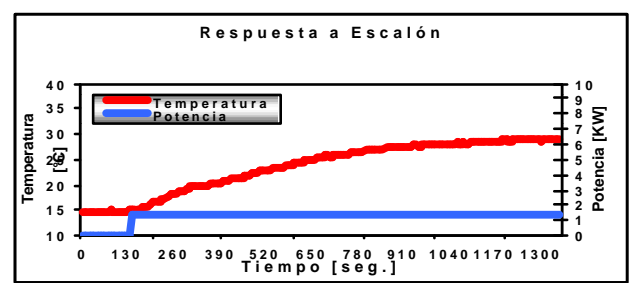

Fig. 2.- Respuesta a Escalón, $\Delta \mathrm{u}(\mathrm{t})=1.43[\mathrm{KW}]$,

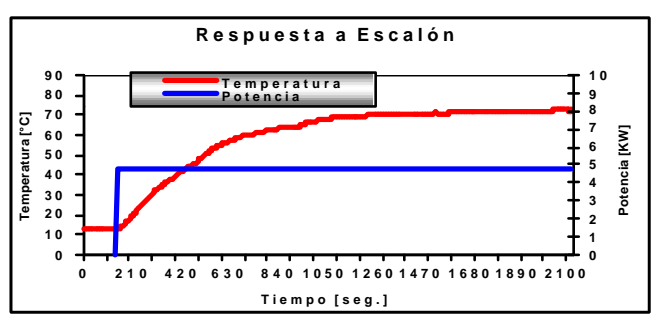

Fig. 3.- Respuesta a Escalón, $\Delta \mathrm{u}(\mathrm{t})=4.76[\mathrm{KW}]$ 


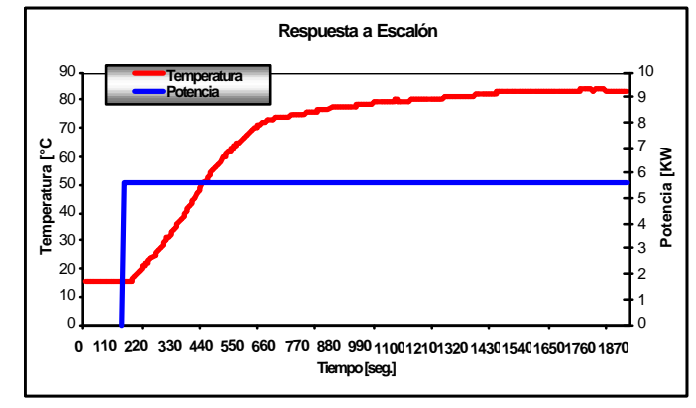

Fig. 4.- Respuesta a Escalón, $\Delta \mathrm{u}(\mathrm{t})=5.60 \mathrm{~W}]$

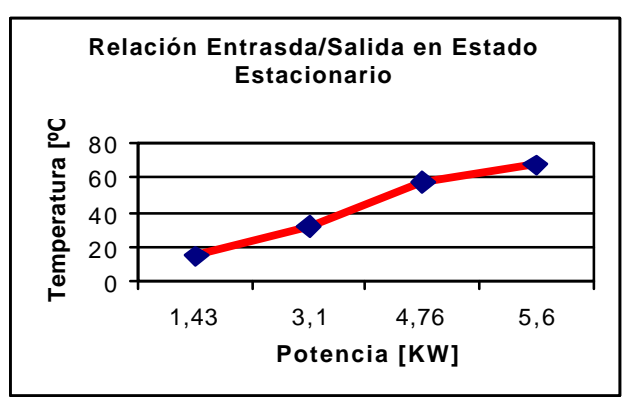

Fig. 5.- Relación entrada /salida en estado de régimen estacionario

\section{CONTROLADOR DIFUSO PROPORCIONAL INTEGRAL (CDPI)}

El controlador difuso PI más difundido, es sin duda, el propuesto por Mac Vicar-Whelan [5], el cual emula un controlador PI estándar (ver Fig. 6). Básicamente todos los controladores difusos mantienen una estructura similar, sin embargo, las diferencias entre ellos son: el método de inferencia, la base del conocimiento y el método de defusificación.

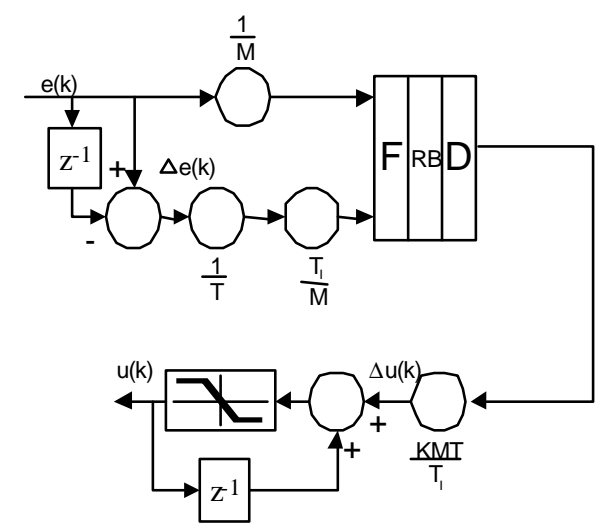

Fig. 6.- Esquema del controlador difuso proporcional integral "CDPI"
El error realimentado $e$, es

$$
e=T_{\text {ref }}-T_{\text {lecho }}
$$

donde $T_{r e f}$ es la temperatura deseada del lecho y $T_{\text {lecho }}$ es la temperatura al interior del lecho. La salida del controlador comanda la potencia de los calefactores.

Al igual que en [3] se probaron diferentes cantidades de conjuntos difusos, y empíricamente se observó que 7 conjunto difusos para el error y 7 conjuntos difusos para el incremento del error presentaban el mejor desempeño. En consecuencia da como resultado 49 reglas difusas. Las funciones de pertenencia se presentan en la figura 7 nominadas Negativo Grande (NG), Negativo Mediano (NM), Negativo pequeño (NP), cero (CE), Positivo Pequeño (PP), Positivo Mediano (PM) y Positivo Grande (PG) y las reglas difusas respectivas se muestran en la Tabla 1.

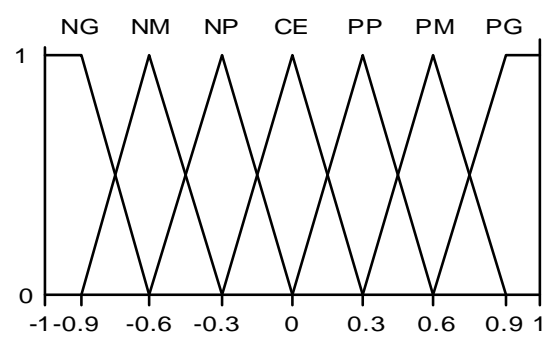

Fig. 7.- Conjuntos difusos para el error y el incremento del error

Tabla 1.- Reglas difusas del controlador PI difuso (CDPI)

\begin{tabular}{|c|c|c|c|c|c|c|c|}
\hline$\Delta_{e(k)}(k)$ & $N G$ & $N M$ & $N P$ & $C E$ & $P P$ & $P M$ & $P G$ \\
\hline$P G$ & CE & PP & PM & PG & PG & PG & PG \\
\hline PM & NP & CE & PP & PM & PG & PG & PG \\
\hline PP & NM & NP & CE & PP & PM & PG & PG \\
\hline CE & NG & NM & NP & CE & PP & PM & PG \\
\hline NP & NG & NG & NM & NP & CE & PP & PM \\
\hline NM & NG & NG & NG & NM & NP & CE & PP \\
\hline NG & NG & NG & NG & NG & NM & NP & CE \\
\hline
\end{tabular}

\section{CONTROLADOR DIFUSO PROPORCIONAI PREALIMENTADO (CDPP)}

El CDPP se muestra en la Fig. 8, donde se combina un lazo de control proporcional más un control prealimentado. Dado que la no linealidad en la temperatura de referencia incide en forma importante 
sobre la dinámica del proceso, se prealimenta esta variable.

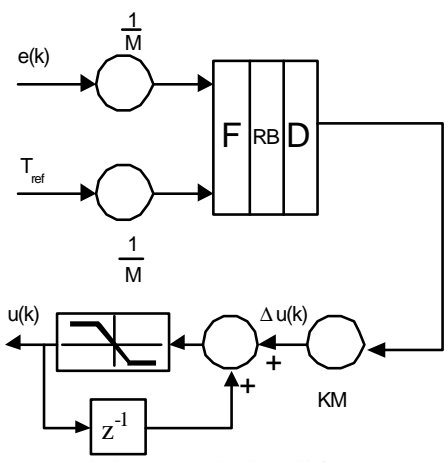

Fig. 8.- Esquema del controlador difuso proporcional prealimentado CDPP

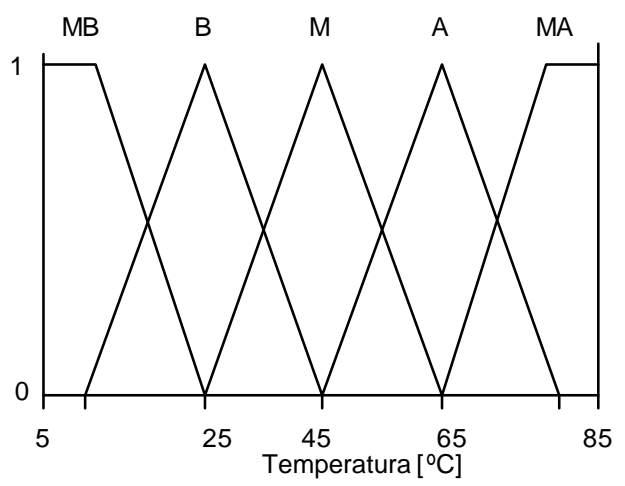

Fig. 9.- Conjuntos difusos para la referencia

En total el controlador esta conformado por 35 reglas difusas. Es así como el error esta conformado por las siete funciones de pertenencia de la figura 7. La temperatura de referencia está asociada con 5 funciones de pertenencia (ver figura 9): Muy Bajo (MB), Bajo (B), Medio (M), Alto (A) y Muy Alto (MA). Las 35 reglas difusas resultantes activan 7 "singletons" denominados: Mucho Más Bajo (MMB), Muy Bajo (MB), Bajo (B), Medio (M), Alto (A), Muy Alto (MA) y Mucho Más Alto (MMA).

Tabla 2.- Reglas difusas del controlador PP difuso (CDPP)

\begin{tabular}{|c|c|c|l|l|c|c|c|}
\hline MB & MMB & MMB & MB & MB & B & A & MMA \\
\hline B & MMB & MB & MB & B & M & MA & MMA \\
\hline M & MMB & MB & B & M & A & MA & MMA \\
\hline A & MMB & MB & M & A & MA & MA & MMA \\
\hline MA & MMB & B & A & MA & MA & MMA & MMA \\
\hline
\end{tabular}

\section{RESULTADOS}

Se efectuaron una variedad de pruebas de laboratorio para destacar las ventajas del controlador CDPP con respecto al CDPI. De acuerdo a las exigencias de los especialistas en el manejo de secado, las pruebas realizadas fueron para diferentes tipos de carga de afrechos, distintas humedades del afrecho y distintas obstrucciones de aire.

Dos experimentos representativos se escogieron para comparar los controladores en discusión.

La fig.10.- muestra la variación de temperatura bajo control CDPI y CDPP cuando es aplicada una señal escalón en la referencia de temperatura. Como puede observarse, la respuesta del CDPP es más rápida y tiene menos fluctuaciones que el CDPI. Es así como a los 290 seg. (4.8 min.) el CDPP alcanza la referencia, en cambio el CDPI la alcanza a los $570 \mathrm{seg}(9.5 \mathrm{~min})$, o sea aproximadamente el doble de tiemp o.

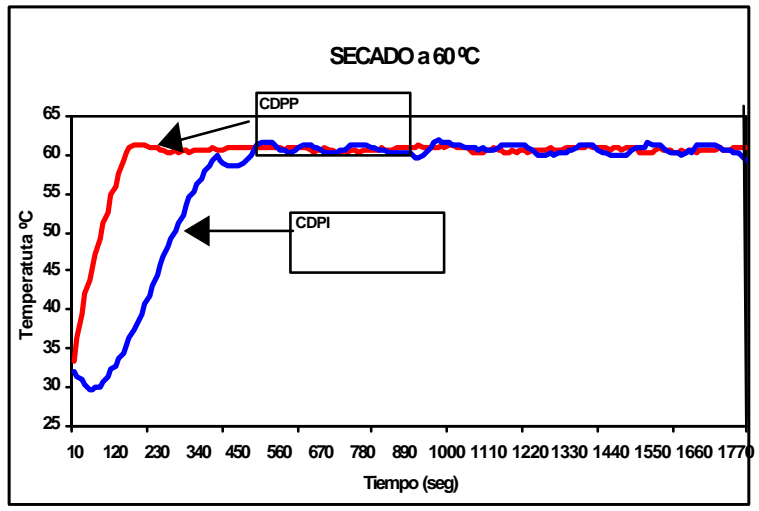

Fig. 10.- Respuesta de la variable controlada frente a un escalón de Temperatura en la referencia de $60^{\circ} \mathrm{C}$

En la Fig. 11 se aplica un escalón en la temperatura de referencia de $80{ }^{\circ} \mathrm{C}$. Aquí se aprecia también un ajuste mas rápido y mejor de la temperatura del lecho a la referencia de temperatura en el CDPP.

En la Tabla 3 se muestra la comparación de ambos controladores considerando índices como el máximo overshoot, el tiempo de crecimiento, el tiempo de asentamiento y el tiempo de peak. De la tabla podemos observar que el CDPP tiene un mejor desempeño en todos los casos. 


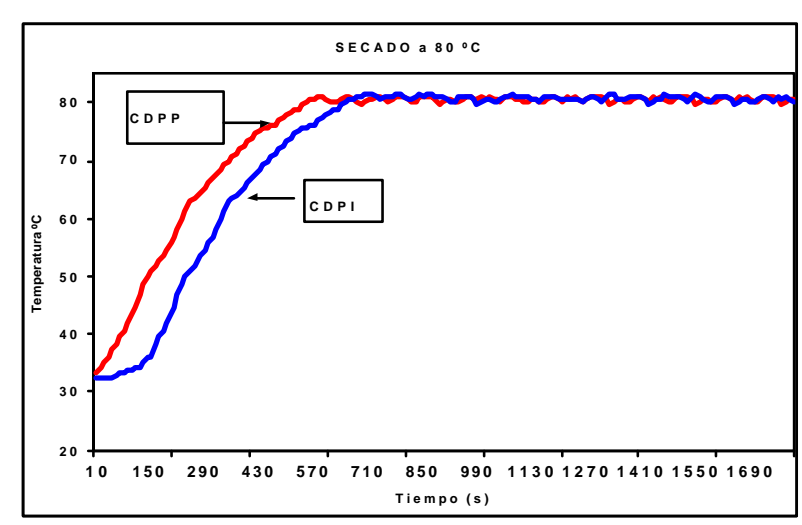

Fig. 11.- Respuesta de la variable controlada frente a un escalón de temperatura de referencia de $80{ }^{\circ} \mathrm{C}$

Tabla 3.- Comparación de controladores difusos PIy PP

\begin{tabular}{|c|c|c|c|c|c|cc|c|}
\hline Ref. & Overshoot & $\begin{array}{c}\text { Tiempo de } \\
\text { Crecimiento } \\
{[\mathrm{seg}]}\end{array}$ & $\begin{array}{c}\text { Tiempo de } \\
\text { Asentamient o } \\
{[\mathrm{seg}]}\end{array}$ & $\begin{array}{c}\text { Tiempo de } \\
\text { Peak } \\
{[\mathrm{seg}]}\end{array}$ \\
\hline$\left.{ }^{\circ} \mathrm{C}\right]$ & \multicolumn{2}{|c|}{$[\%]$} & $\mathrm{PI}$ & $\mathrm{PP}$ & $\mathrm{PI}$ & $\mathrm{PP}$ & $\mathrm{PI}$ & $\mathrm{PP}$ \\
\hline & $\mathrm{PI}$ & $\mathrm{PP}$ & & 150 & 730 & 580 & 440 & 270 \\
\hline 40 & 4.5 & 2.7 & 190 & 150 & 270 & 260 & 410 & 190 \\
60 & 3.2 & 2.1 & 320 & 140 & 610 & & \\
\hline 80 & 1.2 & 1.2 & 350 & 340 & 740 & 620 & 650 & 580 \\
\hline
\end{tabular}

\section{CONCLUSIONES}

En este trabajo se desarrollaron dos algoritmos de control difuso para controlar la temperatura de un secador de lecho fluidizado a escala piloto. Ambos algoritmos mostraron un buen desempeño ante condiciones de operación reales. En el algoritmo de Control Difuso Proporcional Prealimentado se incorporó una prealimentación difusa de la referencia con un notorio mejoramiento con respecto al Control Difuso tipo PI. Como trabajo futuro queda abierta la posibilidad de seguir probando otras estrategias de control que incorpore un esquema adaptivo en el seguimiento no lineal de la referencia o aplicar un controlador predictivo difuso.

\section{AGRADECIMIENTOS}

Los autores agradecen al proyecto Diufro 120323 que ha hecho posible la realización de este trabajo.

\section{REFERENCIAS}

[1] L.A. Ochoa Martínez, S. Machuca Rosales, L. Montoya Ayón y A. Pérez Ricardez; "Performance of an unheated fluidised bed dryer". Engineering $\&$ Food at 7 th International Congress on Engineering and Food, 1997.

[2] L. A. Ochoa Martínez, S. Machuca Rosales, Montoya Ayón L. y A. Pérez Ricardez, "Performance of a Fluidesed Bed Dryer Operating at Low Temperature"; UBAMARI, $\mathrm{N}^{\circ}$ 39, 3-9, ISSN 0185-4518, 1996.

[3] C. Haissig; "Adaptive Fuzzy Temperature Control for Hydronic Heating Systems" IEEE Control System, vol.20,no 2, pp. 38-48, April. 2000.

[4] M. Sugeno; "Theory of Fuzzy Integrals and its application," Ph.D. dissertation, Tokyo Ins. Technol., Japan, 1974.

[5] C. Lee, "Fuzzy logic in control systems: Fuzzy logic controller, part I", IEEE Trans. Systems, Man, Cybernet, vol. 20, no. 2, pp. 404-418, 1990.

[6] J. Medina y J. Pavez; "Automatización de la Planta Piloto de Secado" del Instituto de agroindustria". Trabajo de título para la obtención del Título Profesional de Ingeniero de Ejecución Electrónica. Temuco, Chile. 1999.

[7] P.J. Mac Vicar Whelan; "Fuzzy Set for manmachine interaction”, Int. J. Man Machine Studies, Vol. 8, pp. 687-697, Nov. 1976. 